\title{
Real-World Reductions in Oral Corticosteroid Use in the USA Following Mepolizumab Therapy for Severe Asthma
}

This article was published in the following Dove Press journal: Journal of Asthma and Allergy

\author{
Jared Silver $\mathbb{D}^{\prime}$ \\ Michael Bogart' \\ Elizabeth Packnett ${ }^{2}$ \\ Juan $\mathrm{Wu}^{2}$ \\ Donna McMorrow ${ }^{2}$ \\ Beth Hahn (iD) \\ 'US Medical Affairs, GSK, Research \\ Triangle Park, NC, USA; ${ }^{2}$ Life Sciences, \\ IBM Watson Health, Bethesda, MD, USA
}

Background: Patients with severe asthma often require oral corticosteroid (OCS) treatment. Clinical trials have demonstrated that mepolizumab can reduce OCS dependence, but realworld data are limited.

Objective: To evaluate the impact of mepolizumab on OCS use, asthma exacerbations, and asthma exacerbation-related costs in a real-world setting.

Methods: This retrospective cohort study (GSK ID: 209642; HO-19-19597) analyzed data from the MarketScan ${ }^{\circledR}$ Commercial database (identification period: November 2015September 2017). Patients were $\geq 12$ years old at mepolizumab initiation (index date), had a baseline asthma diagnosis, and received $\geq 2$ mepolizumab administrations in the first 6 months of follow-up. OCS use, asthma exacerbation rate, and asthma exacerbation-related costs were assessed in the 12-months before (baseline) and 12-months after (follow-up) mepolizumab initiation.

Results: Mepolizumab was associated with a $14.7 \%$ reduction in the proportion of patients with $\geq 1$ OCS claim from baseline to follow-up $(93.4 \%$ vs $79.7 \% ; P<0.001)$. The mean numbers of OCS claims/patient and OCS bursts ( $\geq 20 \mathrm{mg}$ prednisone equivalents for 3-28 days) between baseline and follow-up were also reduced by $29.1 \%(P<0.001)$ and $36.8 \%$ $(P<0.001)$, respectively. Reductions in chronic OCS use were demonstrated during follow-up in patients with baseline mean OCS dose $\geq 5 \mathrm{mg}$ and those with a mean OCS dose $\geq 10 \mathrm{mg} 90$ days before index; the proportion of patients with no OCS use also increased from $6.6 \%$ to $20.3 \%$ between baseline and follow-up.

Conclusion: Our findings demonstrate that mepolizumab therapy is associated with reduced OCS use in patients treated in a real-world setting, potentially mitigating adverse health sequelae caused by OCS use in these patients.

Keywords: severe asthma, mepolizumab, claims, real-world study, oral corticosteroids, healthcare resource utilization

\section{Plain Language Summary}

Why was the study done?

Oral corticosteroid use in patients with severe asthma has been linked to numerous adverse health effects. Clinical trials have shown that mepolizumab therapy can reduce dependence on oral corticosteroids. However, data from real-world non-clinical trial settings are limited.

What did the researchers do and find?

To see if mepolizumab therapy can reduce dependence on oral corticosteroids in a realworld situation, data from a commercial US healthcare claims database were analyzed. The 
study found that mepolizumab therapy was associated with reduced oral corticosteroid use, reduced asthma exacerbations, and exacerbation-related healthcare resource utilization and costs in patients with severe asthma.

\section{What do these results mean?}

These results support mepolizumab clinical trial findings, providing further information for physicians to enable them to make treatment decisions for patients with severe asthma.

\section{Introduction}

Severe asthma is defined as asthma that requires maximal, optimized inhaled corticosteroid (ICS) therapy plus another controller to remain under control, or which remains uncontrolled despite this therapy. ${ }^{1,2}$ It is estimated to affect around $3-10 \%$ of the general asthma population, ${ }^{1,2}$ and patients with severe asthma have an impaired quality of life and higher healthcare resource utilization (HCRU) and costs when compared with patients with non-severe asthma. ${ }^{3,4}$ An elevated risk of mortality has also been demonstrated in adults with severe asthma. ${ }^{5}$ Several distinct phenotypes of severe asthma have been described, one of which is severe eosinophilic asthma, characterized by persistent eosinophilic inflammation, frequent exacerbations, and a dependence on oral corticosteroids (OCS) to achieve asthma control. ${ }^{6}$

Although not all patients with severe asthma will be prescribed OCS medications, OCS use is high in this population, and has been associated with numerous severe/adverse health sequelae. Therefore, physicians typically strive to reduce the dosage. ${ }^{7,8}$ Several biologic therapies have been developed for the treatment of severe asthma, which have been shown to have OCS-sparing effects. ${ }^{9}$ One such biologic is mepolizumab, a targeted, humanized anti-interleukin-5 monoclonal antibody, which is approved for use in patients with severe eosinophilic asthma and eosinophilic granulomatosis with polyangiitis (EGPA). ${ }^{10}$ Results from clinical trials in patients with severe eosinophilic asthma have shown that mepolizumab therapy reduces annual exacerbation rates and dependence on OCS, and improves asthma control, lung function and health-related quality of life compared with placebo. ${ }^{11-14}$ Initial evidence from several real-world studies have also demonstrated that mepolizumab therapy in these individuals reduces OCS use and exacerbation rates compared with placebo; however, interpretation is limited by small sample sizes and limited assessment of OCS use. ${ }^{15-18}$

The aim of this study was to build on the findings from previous real-world studies and comprehensively examine the impact of mepolizumab treatment initiation on OCS use, rate of asthma exacerbations and asthma exacerbation-related HCRU and costs in patients with severe asthma using data from a large commercial US healthcare claims database.

\section{Methods}

\section{Study Design}

This was a retrospective cohort study of patients with asthma treated with mepolizumab using data from the IBM Watson Health MarketScan ${ }^{\circledR}$ Commercial and Encounters Database (GSK ID: 209642; HO-19-19597). Patients treated with mepolizumab were identified between November 1, 2015 and September 30, 2017 (identification period); the date of the first medical or pharmacy claim for mepolizumab was defined as the index date (Figure 1). Data were collected during the baseline period (12 months prior to and including the index date) and during the follow-up period (12 months following the index date).

\section{Data Source}

The MarketScan ${ }^{\circledR}$ Commercial Claims and Encounters Database contains the inpatient, outpatient, and outpatient prescription drug experience of approximately 147.9 million patients with employer-sponsored health insurance (including their dependents) from all US census regions, covered under a variety of fee-for-service and capitated health plans, including exclusive provider organizations, preferred provider organizations, point of service plans, indemnity plans, and health maintenance organizations. Detailed cost, use and outcomes data for healthcare services performed in

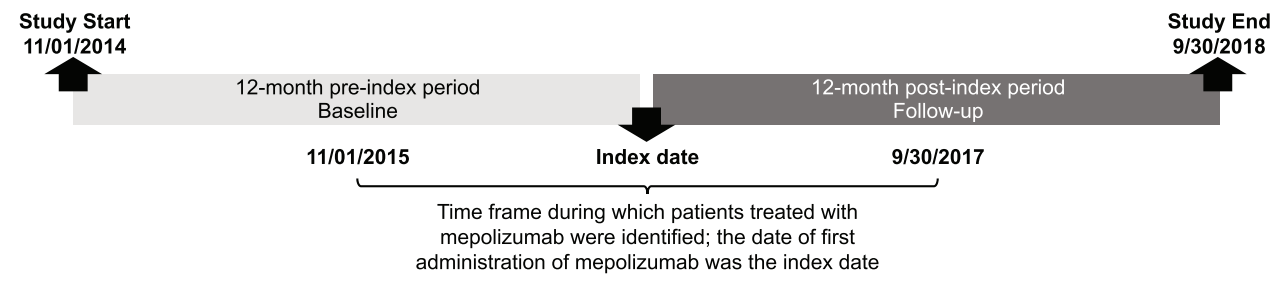

Figure I Study design. 
both inpatient and outpatient settings are recorded in the database. Laboratory data such as blood eosinophil counts, lung function measurements, and imaging data were only available for a subset of patients within this database so were not included in this study. The overall age distribution of the MarketScan ${ }^{\circledR}$ Commercial Claims and Encounters Database population is similar to that of a nationally representative population of persons with employer-sponsored health insurance in the Medical Expenditure Panel Survey.

\section{Patients}

Eligible patients were $\geq 12$ years of age on the index date and received mepolizumab during the identification period. Evidence of mepolizumab treatment was determined using the healthcare common procedure coding system (HCPCS) codes C9473, J2182 on a medical claim or the national drug code (NDC) 00173-0881-01 on a pharmacy claim. Patients also had to have continuous enrolment with medical and pharmacy benefits during the baseline and follow-up periods, $\geq 1$ asthma diagnosis with an International Classification of Diseases (ICD) 9th or 10th revision diagnosis code for asthma (ICD-9: 493.xx, ICD-10: J45.xx) in any position on a medical claim, excluding claims billed for testing or diagnostic purposes during the 12-month baseline period, $\geq 2$ mepolizumab administrations during the first 6 months of the follow-up period. Patients with evidence of mepolizumab use during the 12-month baseline period or evidence of omalizumab, reslizumab, benralizumab, or dupilumab use (as assessed by HCPCS codes or NDCs) during the 12-month baseline or follow-up periods were excluded from the analysis.

\section{Endpoints and Assessments}

The primary outcome measure was OCS use during the 12 months prior to mepolizumab administration relative to the 12 months after mepolizumab administration. OCS use was assessed using HCPCS codes or the NDC. As there is no agreed-upon single measure of OCS use, several measures were assessed. The two main primary endpoints were the change in percentage of patients with any OCS use during the 12-month baseline versus follow-up periods and the change in mean number of OCS pharmacy claims per patient during the 12-month baseline versus follow-up periods. Any OCS use was assessed as a binary indicator to determine the percentage of patients who have any OCS use, and mean number of OCS medical or pharmacy claims per patient was described as a continuous variable. The mean number of claims was reported overall, and exacerbation-related OCS claims were reported separately. Intravenous (IV)/intramuscular (IM)/oral CS were used when identifying exacerbations; however, the OCS outcomes were identified using only oral CS.

To better describe the population, the following primary endpoints were also examined: change in OCS bursts, change in chronic OCS use and change in mean daily OCS dose. OCS bursts were assessed as a binary indicator to determine the percentage of patients with $\geq 1$ OCS burst. The total number of OCS bursts were also assessed separately as a continuous variable. OCS bursts were required to meet the following criteria: average daily dose $\geq 20 \mathrm{mg}$ prednisone equivalents for a duration of 3-28 days and one outpatient or emergency room (ER) claim with a diagnosis of asthma (ICD-9: 493.xx, ICD-10: J45.xx) \pm 7 days of the pharmacy claim. Chronic OCS use was measured using two definitions: patients with a mean daily OCS dose (prednisone equivalents) $\geq 5 \mathrm{mg}$ over the 12-month baseline or follow-up period, or $\geq 10 \mathrm{mg}$ in the last 90 days of the baseline period including the index date. Mean daily OCS use was calculated among all patients as prednisone equivalents over each respective 12-month period (ie baseline and follow-up). Analyses were also conducted separately on the subset of patients with any OCS claim during the baseline period.

The secondary endpoints of the study were to evaluate changes in the annualized rate of asthma exacerbations, and asthma exacerbation-related costs for patients receiving mepolizumab in the 12 months prior to mepolizumab administration compared with the 12 months after mepolizumab administration. Exacerbations that required HCRU were identified if either of the following criteria were met: 1) one outpatient or ER claim with a diagnosis of asthma (ICD-9: 493.xx, ICD-10: J45.xx) and $\geq 1$ claim for a systemic corticosteroid (IM, IV, or oral) \pm 5 days of the encounter or 2 ) exacerbations resulting in hospitalization defined as inpatient hospital admissions with a diagnosis of asthma (ICD-9: 493. $\mathrm{xx}$, ICD-10: J45.xx) as a primary diagnosis. Exacerbations that began on the index date were considered as baseline exacerbations. Exacerbations with a start date that occurred within the 14 days following the end date of the prior exacerbation episode were combined into a single episode. Asthma exacerbation-related healthcare costs were also measured; these were based on paid amounts of adjudicated claims, including insurer and health plan payments in addition to patient cost-sharing as co-payments, deductibles, and co-insurance. Asthma exacerbation-related costs were identified as inpatient claims with a primary diagnosis of asthma; 
outpatient claims with an asthma diagnosis in any position; or medical or pharmacy claims for systemic corticosteroids, rescue medications, and asthma medications during the exacerbation event. Costs measured on the index date were considered part of the baseline period and excluded those costs associated with mepolizumab acquisition or administration, consistent with the approach taken in other recent studies. ${ }^{18}$ All costs were adjusted for inflation using the Consumer Price Index and standardized to 2018 US dollars.

\section{Statistical Analysis}

The means, standard deviations, and medians were reported for the continuous variables, and the counts and percentages were reported for the dichotomous or categorical variables. McNemar Chi-squared tests were used to evaluate the statistical significance of the differences for dichotomous or categorical variables and paired $t$-tests were used for the comparison of continuous variables. A $P$-value of 0.05 was the maximum $P$-value for which differences were considered statistically significant. Multivariable regression analysis was not performed, based on the estimated size of the study population and the fact that each patient effectively acted as their own control and was observed for a 12-month fixed period before and after mepolizumab initiation.

\section{Results}

\section{Patient Population}

Baseline demographic and clinical characteristics are summarized in Table 1. A total of 527 patients were identified with a mean (standard deviation [SD]) age of 49.4 (11.9) years; the majority were female $(60.7 \%)$. The most common comorbidities were allergic rhinitis (68.9\%), sinusitis (56.5\%; acute sinusitis, $36.1 \%$ and chronic sinusitis, $41.7 \%)$, and respiratory infections (46.5\%).

\section{OCS Use}

During the baseline period $93.4 \%$ of patients had $\geq 1$ OCS claim compared with $79.7 \%(P<0.001)$ during the follow-up period; consequently, the proportion of patients with no OCS use more than tripled from $6.6 \%$ in the baseline period to $20.3 \%$ in the follow-up period (Figure 2). When the analysis was limited to those patients with any OCS claim during the baseline period $(n=492), 82.5 \%$ had an OCS claim in the follow-up period, resulting in a similar proportion $(17.5 \%)$ of the
Table I Patient Baseline Demographic and Clinical Characteristics

\begin{tabular}{|c|c|}
\hline & $\begin{array}{l}\text { Patients } \\
(\mathrm{N}=527)\end{array}$ \\
\hline Age, mean (SD) & $49.4(11.9)$ \\
\hline \multicolumn{2}{|l|}{ Sex, n (\%) } \\
\hline Female & $320(60.7)$ \\
\hline \multicolumn{2}{|l|}{ Geographic region, $\mathbf{n}(\%)$} \\
\hline Northeast & $116(22.0)$ \\
\hline North Central & $123(23.3)$ \\
\hline South & $212(40.2)$ \\
\hline West & $74(\mid 4.0)$ \\
\hline Unknown & $2(0.4)$ \\
\hline \multicolumn{2}{|l|}{ Insurance plan type, n (\%) } \\
\hline $\mathrm{CDHP} / \mathrm{HDHP}$ & $107(20.3)$ \\
\hline Comprehensive/indemnity & $14(2.7)$ \\
\hline EPO/PPO & $303(57.5)$ \\
\hline $\mathrm{HMO}$ & $64(12.1)$ \\
\hline POS/POS with capitation & $32(6.1)$ \\
\hline Other/unknown & $7(1.3)$ \\
\hline \multicolumn{2}{|l|}{ Use of asthma medications,* n (\%) } \\
\hline OCS & $492(93.4)$ \\
\hline ICS & $227(43.1)$ \\
\hline SABA & $433(82.2)$ \\
\hline SAMA & $51(9.7)$ \\
\hline LABA & $21(4.0)$ \\
\hline LAMA & $168(31.9)$ \\
\hline LTRA & $370(70.2)$ \\
\hline ICS/LABA & $426(80.8)$ \\
\hline ICS/LABA/LAMA & $135(25.6)$ \\
\hline Mast cell stabilizers & $4(0.8)$ \\
\hline Methylxanthines & $33(6.3)$ \\
\hline Deyo-Charlson Comorbidity Index, ${ }^{\dagger}$ Mean (SD) & $1.4(0.9)$ \\
\hline \multicolumn{2}{|l|}{ Comorbid conditions, $\mathrm{n}(\%)$} \\
\hline Allergic rhinitis & $363(68.9)$ \\
\hline Atopic dermatitis & $15(2.8)$ \\
\hline Chronic idiopathic urticaria & $5(0.9)$ \\
\hline COPD & $164(31.1)$ \\
\hline Diabetes & $61(11.6)$ \\
\hline Eosinophilic esophagitis & II (2.I) \\
\hline EGPA & $16(3.0)$ \\
\hline GERD & $179(34.0)$ \\
\hline Herpes virus infection & $8(1.5)$ \\
\hline Herpes simplex & $4(0.8)$ \\
\hline Herpes zoster & $4(0.8)$ \\
\hline Hypertension & $180(34.2)$ \\
\hline Nasal polyps & $109(20.7)$ \\
\hline Respiratory infections & $245(46.5)$ \\
\hline Rheumatoid arthritis & $9(1.7)$ \\
\hline Sinusitis & $298(56.5)$ \\
\hline
\end{tabular}

(Continued) 
Table I (Continued).

\begin{tabular}{|l|l|}
\hline & $\begin{array}{l}\text { Patients } \\
(\mathbf{N}=527)\end{array}$ \\
\hline Acute & $190(36.1)$ \\
Chronic & $220(41.7)$ \\
Systemic lupus erythematosus & $3(0.6)$
\end{tabular}

Notes: *Patients with $\geq$ I claim for the indicated medications during the 12-month baseline period; 'Deyo-Charlson Comorbidity Index categorizes comorbidities of patients based on 17 ICD diagnosis codes/disease categories. ${ }^{23}$ Scoring: each comorbidity category has an associated weight (from I to 6), based on the adjusted risk of mortality or resource use, and the sum of all the weights results in a single comorbidity score for a patient. A score of 0 indicates that no comorbidities were found. The higher the score, the more likely the predicted outcome will result in mortality or higher resource use.

Abbreviations: CDHP, consumer-directed health plan; COPD, chronic obstructive pulmonary disease; EGPA, eosinophilic granulomatosis with polyangiitis; EPO, exclusive provider organization; GERD, gastroesophageal reflux disease; HDHP, highdeductible health plan; HMO, health maintenance organization; ICD, International Classification of Diseases; ICS, inhaled corticosteroids; LABA, long-acting $\beta_{2}$-agonist; LAMA, long-acting muscarinic antagonist; LTRA, leukotriene receptor antagonist; OCS, oral corticosteroid; POS, point-of-service; PPO, preferred provider organization; SABA, short-acting $\beta_{2}$-agonist; SAMA, short-acting muscarinic antagonist; SD, standard deviation.

population being OCS-free in the follow-up period (data not shown).

The mean number of OCS claims per patient decreased by $29.1 \%$ in the follow-up period compared with the baseline period ( 5.5 vs $3.9, P<0.001$; Figure $3 \mathrm{~A}$ ), and the mean number of OCS bursts (defined as $\geq 20 \mathrm{mg}$ prednisone equivalents for a duration of 3-28 days) decreased by $36.8 \%$ from the baseline period to the follow-up period (1.9 vs $1.2 ; P<0.001$; Figure $3 \mathrm{~B}$ ). There was also a $21.8 \%$ reduction in the proportion of patients with an OCS burst in the baseline period versus follow-up (68.7\% vs $53.7 \%$, $P<0.001)$. Similar results were obtained when the analysis was limited to patients with OCS use at baseline $(n=492$;

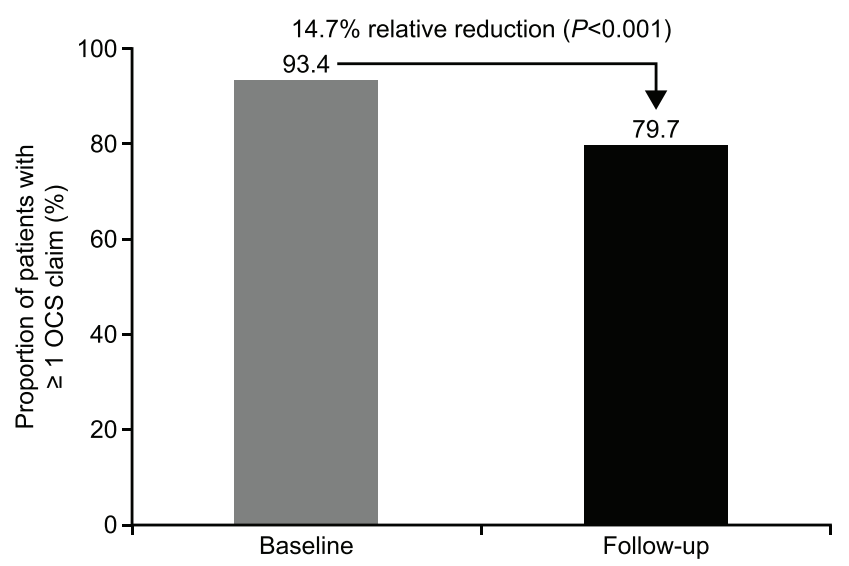

Figure 2 Proportion of patients with any OCS use in the baseline and follow-up periods $(\mathrm{N}=527)$.

Abbreviation: OCS, oral corticosteroid.
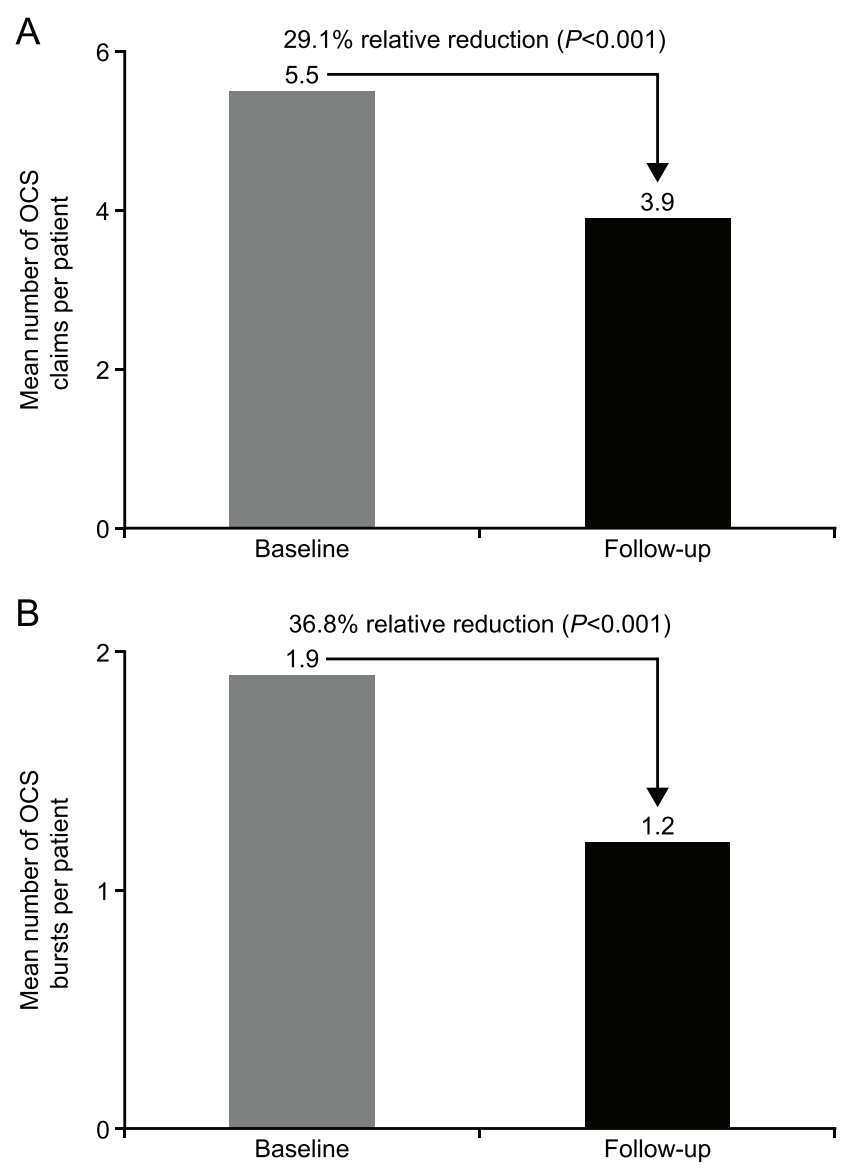

Figure 3 (A) Mean number of OCS claims per patient and (B) mean number of OCS bursts* per patient in the baseline and follow-up periods $(\mathrm{N}=527)$. *OCS burst was defined as meeting the following criteria: average daily dose $\geq 20 \mathrm{mg}$ prednisone equivalents for a duration of 3-28 days and one outpatient or ER claim with a diagnosis of asthma (ICD-9: 493.xx, ICD- I0: J45.xx) \pm 7 days of the pharmacy claim.

Abbreviation: OCS, oral corticosteroid.

relative reductions of $30.5 \%$ and $35.0 \%$ in the mean number of OCS claims and mean number of OCS bursts, respectively, data not shown).

The proportion of patients with chronic OCS use (as measured by a mean daily dose of $\geq 5 \mathrm{mg}$ /day prednisone equivalents over the 12-month baseline or follow-up period) was lower in the follow-up period compared with the baseline period with a reduction of $34.7 \%$ (42.1\% vs $27.5 \%$, $P<0.001$; Figure 4A). Among patients with chronic OCS use $(\geq 10 \mathrm{mg}$ dose prednisone equivalents in the last 90 days of the baseline period; $n=106$ ), the proportion of patients during the follow-up period decreased steadily to 21 patients, a relative reduction of $80.2 \%$, in the final 3 months of the follow-up period (Figure 4B). In total, the mean (SD) daily OCS dose in the last 90 days of the baseline period for the 106 patients with chronic OCS use (defined by $\geq 10 \mathrm{mg}$ dose prednisone equivalents in the last 90 days of the baseline period), was 44 (161) mg/day. Across the entire 


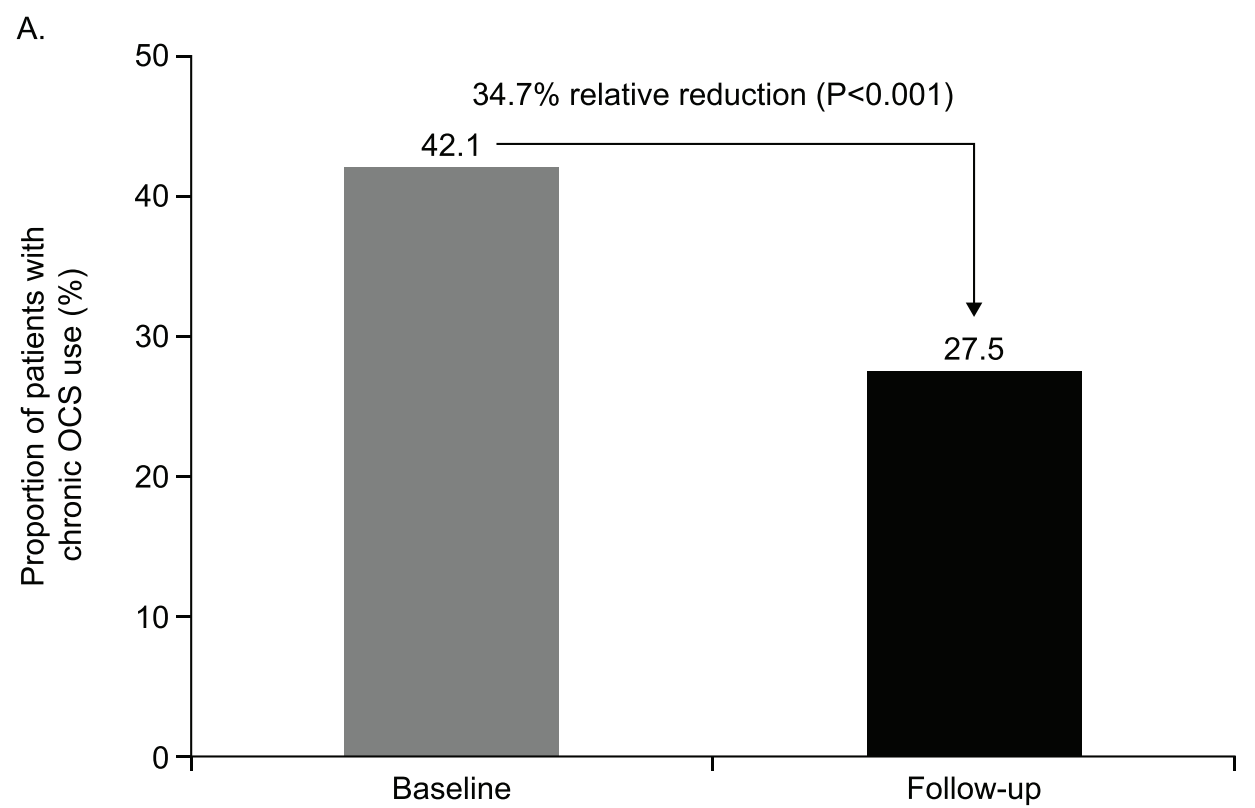

B.

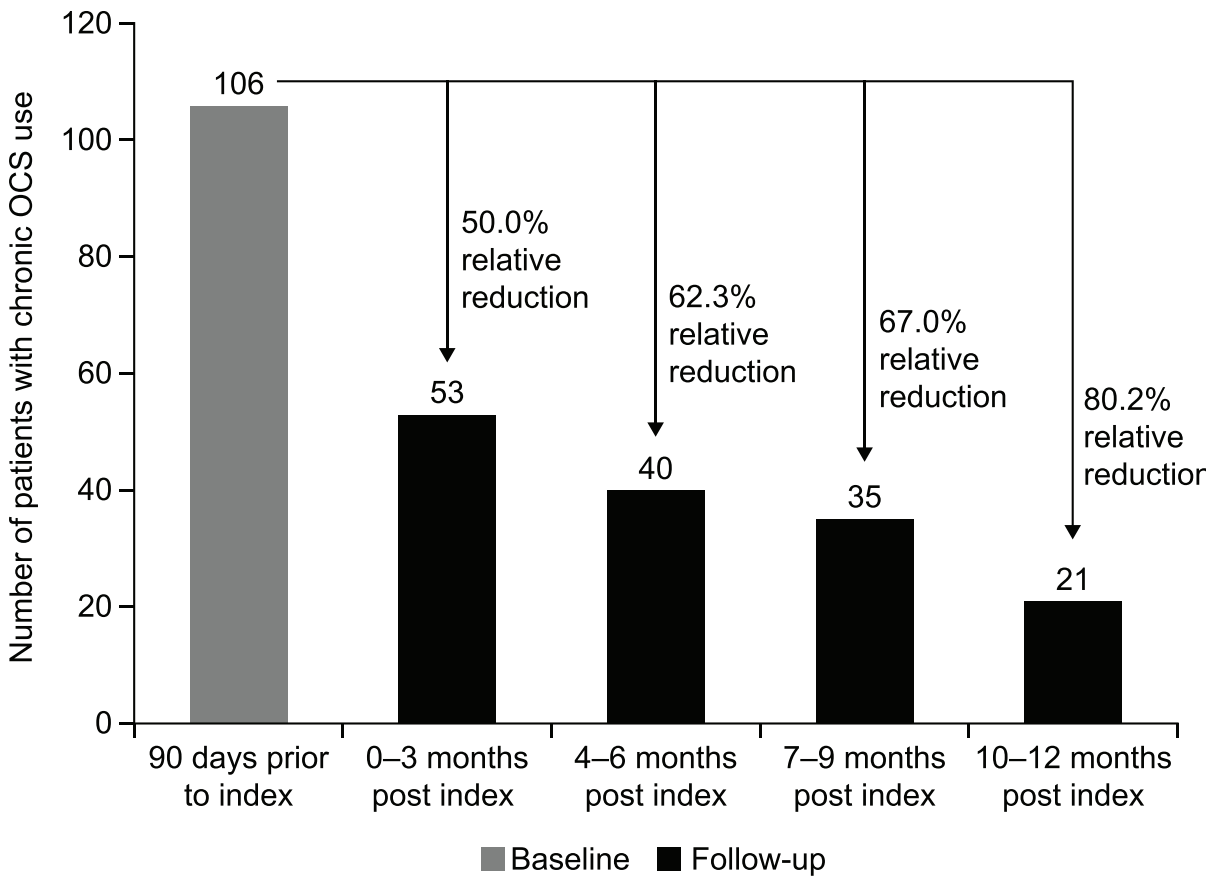

Figure 4 (A) Proportion of patients with chronic OCS use ( $\geq 5 \mathrm{mg} /$ day) in the baseline and follow-up periods ( $\mathrm{N}=527)$ and $(\mathbf{B})$ number of patients with chronic OCS use $(\geq 10 \mathrm{mg} /$ day prednisone equivalents) during the last 90 days of the baseline period $(n=106)$ and analyzed by quarter in the follow-up period. Abbreviation: OCS, oral corticosteroid.

population $(\mathrm{n}=527)$, the mean daily OCS dose decreased from $6.2(7.7) \mathrm{mg}$ /day at baseline to $4.2(6.8) \mathrm{mg} /$ day at follow-up $(P<0.001)$, a reduction of $32.3 \%$. Among all patients with baseline OCS use ( $\mathrm{n}=492), 74.4 \%$ of patients had a reduction in OCS use (Figure 5A) and of these, 63.4\% patients had a reduction $\geq 50 \%$ from baseline to follow-up. Among patients with baseline OCS use, the mean daily OCS dose decreased from $6.7(7.8) \mathrm{mg} /$ day at baseline to 4.5
(6.9) $\mathrm{mg} /$ day at follow-up $(P<0.001)$, a relative reduction of $33.2 \%$. The distribution of the percent reduction among patients with a reduction in OCS dosage is shown in Figure 5B.

\section{Asthma Exacerbations}

The proportion of patients who experienced any asthma exacerbations decreased when comparing the follow-up 
A.

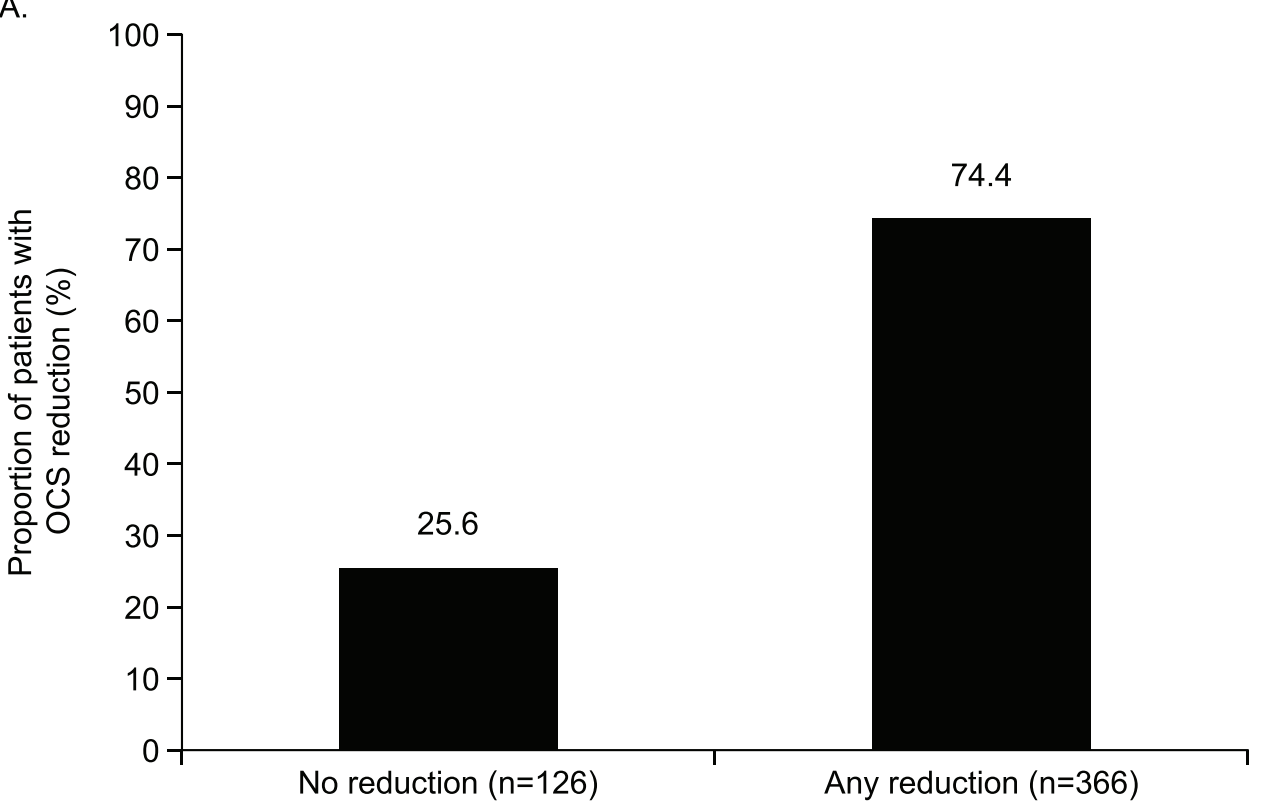

B.

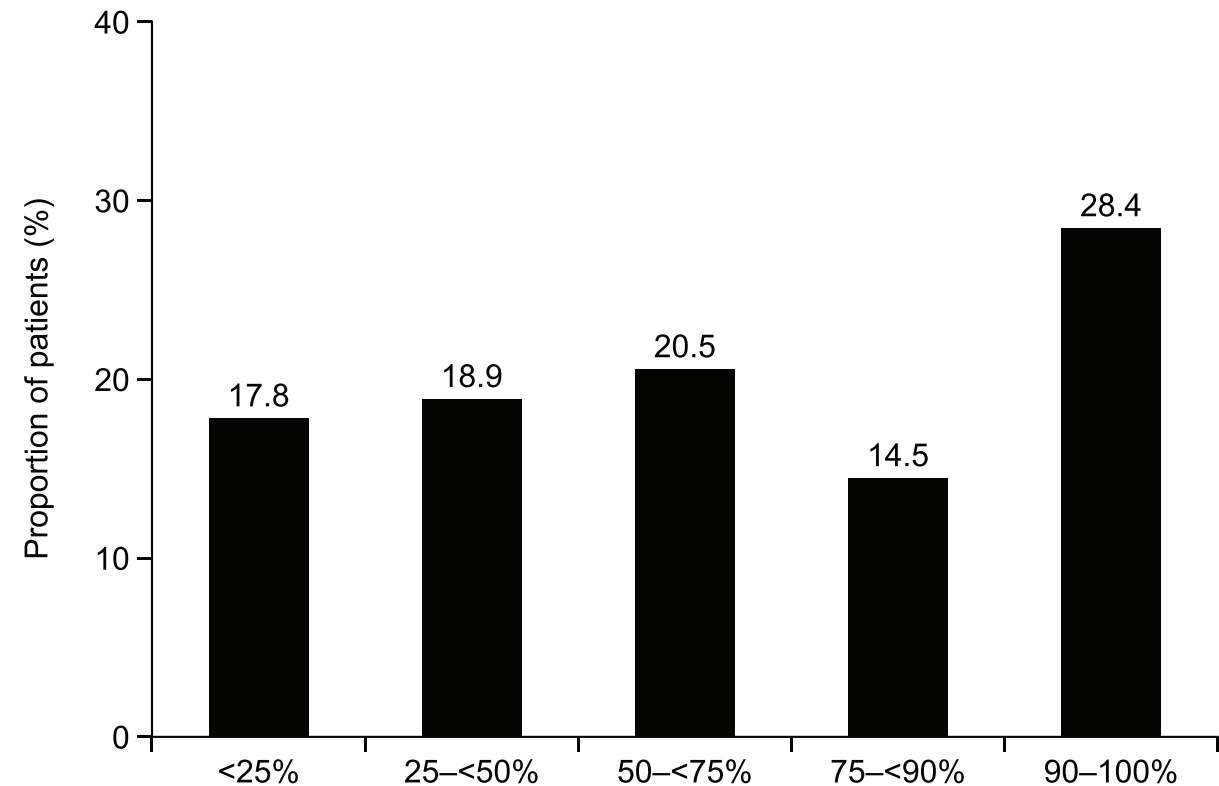

Reduction in mean daily OCS dose
$\mathrm{n}=$
65
69
75
53
104

Figure 5 (A) Proportion of patients with a percentage reduction in the mean daily OCS dose* from baseline to follow-up $(n=492)$ and $(\mathbf{B})$ proportion of patients with a percentage reduction in the mean daily OCS dose among patients with a reduction from baseline to follow-up $(n=366)$. *Mean daily dose was calculated among all patients as prednisone equivalents over the total days prescribed for each respective 12-month period (ie baseline and follow-up). Daily dose >99th percentile was set to 99 th percentile. Abbreviation: OCS, oral corticosteroid.

period with the baseline period $(59.4 \%$ vs $82.5 \%$; $P<0.001)$; the rate of any exacerbations per patient also decreased by $45.5 \%$ in the follow-up period $(1.2$ vs $2.2 ; \quad P<0.001 ;$ Figure S1A). The proportion of patients who experienced an exacerbation resulting in hospitalization decreased in the follow-up period, compared with baseline $(3.2 \%$ vs $7.8 \% ; P<0.001)$; the annualized rate of exacerbations resulting in hospitalization was also reduced by $60.0 \%$ in the follow-up period compared with the baseline period (0.04 vs 0.1 $P<0.001)$ when comparing the baseline period with the follow-up period (Figure S1B). 


\section{Asthma-Exacerbation Related Healthcare Costs}

The total mean asthma exacerbation-related healthcare costs per patient were $51.5 \%$ lower in the follow-up period compared with the baseline period ( $\$ 2219$ vs $\$ 4575 ; P<0.05$; Figure S2). Mean inpatient and outpatient prescription asthma exacerbation-related costs per patient decreased by $55.9 \%$ and $35.0 \%$ in the follow-up period compared with the baseline period ( $\$ 697$ vs $\$ 1582$ and $\$ 464$ vs $\$ 714$, respectively). In addition, the mean total outpatient asthma exacerbation-related costs per patient was reduced by $53.6 \%$ in the follow-up period compared with baseline (\$1058 vs \$2279).

\section{Discussion}

In this study, we assessed the real-world impact of mepolizumab treatment on OCS use, rate of asthma exacerbations and asthma exacerbation-related healthcare costs in patients with asthma using data from a large US administrative claims database. Based on the analysis of multiple measures of OCS burden in patients with severe asthma, we found that mepolizumab reduced the use of OCS, including in those individuals who were chronic OCS users. Mepolizumab was also found to reduce the annualized rate of asthma exacerbations and asthma exacerbation-related costs. As such, these data provide further valuable evidence of the effectiveness of mepolizumab in a real-world setting.

Both long- and short-term treatments with corticosteroids are associated with multiple adverse health effects on the psychiatric, neurologic, endocrine, skin/soft tissue, developmental, ophthalmic, immunologic, cardiovascular, gastrointestinal, and musculoskeletal systems that may be severe or life-threatening. ${ }^{719-21}$ As such, corticosteroid dose reduction is an important goal for physicians and it has been suggested that a $50 \%$ reduction in exposure to regular and rescue OCS is a realistic target. ${ }^{22}$ Our results on OCS reduction therefore represent an important clinical benefit for patients treated with mepolizumab, particularly as we found that almost three quarters of patients had a reduction in OCS use following mepolizumab treatment and approximately one-fifth of patients had no OCS use in the follow-up period. Importantly these findings also support data from the Phase III SIRIUS study, where more patients receiving mepolizumab than those receiving placebo had a reduction in OCS dose of $90-100 \%$ (23\% vs $11 \%)$ and a reduction of $75-<90 \%(17 \%$ vs $8 \%$ ) after 20 weeks of treatment. ${ }^{11}$ It is important to note that patients in the SIRIUS study all had severe disease and were receiving optimized OCS treatment (ranging from 5 to $35 \mathrm{mg}$ /day prednisone equivalent) before the initiation of mepolizumab treatment, and a strict OCS reduction phase ( 1.25 to $10 \mathrm{mg}$ /day every 4 weeks) was conducted during the study. ${ }^{11}$ The fact that we observed broadly similar results in a real-world setting highlights the robust OCS-sparing effect associated with mepolizumab and is also consistent with results from several other smaller, real-world studies. ${ }^{15-18}$

We also found that mepolizumab treatment was associated with a $45.5 \%$ reduction in the annualized rate of asthma exacerbations and $60 \%$ reduction in the annualized rate of exacerbations resulting in hospitalization. The reductions we observed are broadly in line with those reported in smaller real-world studies, ${ }^{15-18}$ and with results from the SIRIUS study where, despite receiving a reduced OCS dose, patients in the mepolizumab group had a relative reduction of $32 \%$ in the annualized rate of exacerbations compared with those in the placebo group. ${ }^{11}$ Our data also support the efficacy of mepolizumab observed in other clinical trials (using the licensed dose, $100 \mathrm{mg}$ subcutaneously), where reductions of $53-58 \%$ in the rate of all exacerbations versus placebo and $69 \%$ in the rate of exacerbations requiring hospitalization versus placebo were reported. ${ }^{13,14}$ In our study, the reductions in exacerbation frequency were not as dramatic as those reported in these pivotal clinical trials. ${ }^{13,14}$ This may be explained by differences in disease severity in the different study populations and the fact that during a clinical trial, patients are strictly monitored and receive all doses of therapy, whereas in real-world clinical practice, patients may not have complete adherence to therapy. Indeed, although mepolizumab is recommended for administration once every 4 weeks, the mean number of doses received in this study was 10.3 during the 12 -month follow-up period, with $35.9 \%$ of patients receiving fewer than 10 doses. In addition, in a clinical trial, exacerbation data are more rigorously evaluated and tracked, whereas real-world evaluations such as this study are dependent on the data that are collected during routine clinical practice.

We also investigated the impact of mepolizumab on asthma exacerbation-related healthcare costs, and found that both total and individual component costs were significantly reduced following mepolizumab treatment. Llanos et al also reported significant reductions in asthma exacerbation-related costs following mepolizumab costs, albeit in a smaller patient population $(\mathrm{n}=346) .{ }^{18}$ Therefore, our findings in a larger patient population not only provide evidence of the benefit of mepolizumab in reducing disease symptoms, but also indicate that mepolizumab 
may help to reduce the HCRU and costs associated with treating asthma exacerbations.

Several limitations should be considered when interpreting our results. First, as this was a real-world study, there was no placebo-control group, although each patient's data from the baseline period were used as their own control after mepolizumab initiation. This study design is limited by possible time-varying confounding such as changes in individual characteristics or changes in concomitant medication over time, although this is more of an issue in studies conducted over periods longer than the 2-year assessment used for this study. Second, the study population included patients with private commercial insurance in the USA, and as such the results of this analysis may not be generalizable to patients with asthma based in other countries or those with other types of health insurance (such as Medicaid, Medicare) or no insurance. In particular, the data in this study may reflect a 'healthier' patient population compared with the entire US population, as many of patients included in the MarketScan ${ }^{\circledR}$ database were employed. Third, claims databases rely on administrative claims data for clinical detail and therefore do not provide the same level of detail as a clinical trial. Administrative data are also subject to coding limitations and data entry error. However, because provider reimbursement requires the submission of accurate data on medical claims, such errors are likely to be infrequent if they do occur. Fourth, data regarding medications that were administered during an inpatient admission were not captured in the database and may have led to an underestimation of the total number of patients who received asthma treatments as well as an underestimation of steroid exposure, although these limitations may be mitigated by factors such as low hospitalization rates particularly after treatment. Finally, the study design assumed that there was no disease progression and that exacerbation rates would remain stable, irrespective of treatment. Despite these limitations, this real-world study provides valuable insights for healthcare professionals into the impact of mepolizumab treatment outside the controlled environment of a clinical trial and supports findings from clinical trials with mepolizumab. This data may help inform the healthcare professional plan of care with usage of mepolizumab by offering insights into the potential degree of reduction in OCS exposure over time for their patients. Such insights may help guide clinical decision-making with mepolizumab regarding treatment initiation and subsequent re-evaluation.

\section{Conclusions}

In conclusion, this analysis of data from a real-world population of patients with severe asthma demonstrated a reduction in OCS use, asthma exacerbations and asthma exacerbation-related costs following mepolizumab treatment. These results complement findings from randomized clinical trials and provide further evidence of the clinical benefit of mepolizumab in real-world clinical practice, as well as the potential impact of this therapy in helping to reduce the HCRU and costs associated with treating asthma exacerbations. Use of mepolizumab to aid OCS use reduction may have real-world benefits, helping to mitigate the detrimental effects of these medications commonly prescribed for severe asthma.

\section{Abbreviations}

CDHP, consumer-directed health plan; COPD, chronic obstructive pulmonary disease; CPT, current procedural terminology; EGPA, eosinophilic granulomatosis with polyangiitis; EPO, exclusive provider organization; ER, emergency room; GERD, gastroesophageal reflux disease; HCPCS, healthcare common procedure coding system; HCRU, healthcare resource utilization; HDHP, high-deductible health plan; HMO, health maintenance organization; ICD, international classification of diseases; ICS, inhaled corticosteroids; IM, intramuscular; IV, intravenous; LABA, long-acting $\beta_{2}$-agonist; LAMA, long-acting muscarinic antagonist; LTRA, leukotriene receptor antagonist; NDC, national drug code; OCS, oral corticosteroids; POS, point-of-service, PPO, preferred provider organization; SABA, short-acting $\beta_{2}$-agonist; SAMA, shortacting muscarinic antagonist; $\mathrm{SD}$, standard deviation.

\section{Ethics Approval and Informed Consent}

This study utilized de-identified retrospective claims data, and as such, this study did not require institutional review board review and approval or informed consent.

\section{Consent for Publication}

The authors hereby grant to the journal the license to publish or distribute the manuscript. It is affirmed that the person/s named above are the sole author/s of the manuscript and that neither this manuscript nor portions thereof have been published elsewhere and that this manuscript will not be submitted elsewhere until the journal's editorial process is completed. 


\section{Acknowledgments}

Editorial support (in the form of writing assistance, including development of the initial draft based on author direction, assembling tables and figures, collating authors' comments, grammatical editing, and referencing) was provided by Kerry Knight, PhD, at Fishawack Indicia Ltd, UK, and was funded by GSK. The abstract of this study was presented at the American Thoracic Society (ATS) 2020 virtual conference as a poster presentation. The poster's abstract was published in Am J Respir Crit Care Med: https://www.atsjournals.org/doi/pdf/10.1164/ajrccmconference.2020.201.1_MeetingAbstracts.A7741

\section{Author Contributions}

All authors made a significant contribution to the work reported, whether that is in the conception, study design, execution, acquisition of data, analysis and interpretation, or in all these areas; took part in drafting, revising or critically reviewing the article; gave final approval of the version to be published; have agreed on the journal to which the article has been submitted; and agree to be accountable for all aspects of the work.

\section{Funding}

This study was funded by GlaxoSmithKline (GSK ID: 209642; HO-19-19597).

\section{Disclosure}

JS, MB, and BH are GSK employees and hold stock/share options in GSK. JS was previously and within the past 36 months a full-time employee of Partners Healthcare and Brigham and Women's Hospital. EP, JW, and DM are employees of IBM Watson Health, which received funding from GSK to complete this study. The authors report no other conflicts of interest in this work.

\section{References}

1. GINA. Global initiative for asthma. Global strategy for asthma management and prevention; June 2019. Available from: https://ginasthma. org/wp-content/uploads/2019/06/GINA-2019-main-report-June-2019wms.pdf. Accessed December 2019.

2. Chung KF, Wenzel SE, Brozek JL, et al. International ERS/ATS guidelines on definition, evaluation and treatment of severe asthma. Eur Respir J. 2014;43(2):343-373. doi:10.1183/09031936.00202013

3. Chastek B, Korrer S, Nagar SP, et al. Economic burden of illness among patients with severe asthma in a managed care setting. J Manag Care Spec Pharm. 2016;22(7):848-861.
4. Zeiger RS, Schatz M, Dalal AA, et al. Utilization and costs of severe uncontrolled asthma in a managed-care setting. J Allergy Clin Immunol Pract. 2016;4(1):120-129 e123. doi:10.1016/j.jaip.2015.08.003

5. Omachi TA, Iribarren C, Sarkar U, et al. Risk factors for death in adults with severe asthma. Ann Allergy Asthma Immunol. 2008;101 (2):130-136. doi:10.1016/S1081-1206(10)60200-1

6. Buhl R, Humbert M, Bjermer L, et al. Severe eosinophilic asthma: a roadmap to consensus. Eur Respir J. 2017;49:5. doi:10.1183/ 13993003.00634-2017

7. Sarnes E, Crofford L, Watson M, Dennis G, Kan H, Bass D. Incidence and US costs of corticosteroid-associated adverse events: a systematic literature review. Clin Ther. 2011;33(10):1413-1432. doi:10.1016/j.clinthera.2011.09.009

8. Price DB, Trudo F, Voorham J, et al. Adverse outcomes from initiation of systemic corticosteroids for asthma: long-term observational study. J Asthma Allergy. 2018;11:193-204. doi:10.2147/JAA.S176026

9. Krings JG, McGregor MC, Bacharier LB, Castro M. Biologics for severe asthma: treatment-specific effects are important in choosing a specific agent. J Allergy Clin Immunol Pract. 2019;7(5):1379-1392. doi:10.1016/j.jaip.2019.03.008

10. Nucala GSK. Prescribing information; September 2019. Available from: https://www.gsksource.com/pharma/content/dam/ GlaxoSmithKline/US/en/Prescribing_Information/Nucala/pdf/ NUCALA-PI-PIL.PDF. Accessed December 2019.

11. Bel EH, Wenzel SE, Thompson PJ, et al. Oral glucocorticoid-sparing effect of mepolizumab in eosinophilic asthma. $N$ Engl $J$ Med. 2014;371(13):1189-1197. doi:10.1056/NEJMoa1403291

12. Pavord ID, Korn S, Howarth P, et al. Mepolizumab for severe eosinophilic asthma (DREAM): a multicentre, double-blind, placebo-controlled trial. Lancet. 2012;380(9842):651-659. doi:10.10 16/S0140-6736(12)60988-X

13. Chupp GL, Bradford ES, Albers FC, et al. Efficacy of mepolizumab add-on therapy on health-related quality of life and markers of asthma control in severe eosinophilic asthma (MUSCA): a randomised, double-blind, placebo-controlled, parallel-group, multicentre, phase 3b trial. Lancet Respir Med. 2017;5(5):390-400. doi:10.1016/S2213-2600(17)30125-X

14. Ortega HG, Liu MC, Pavord ID, et al. Mepolizumab treatment in patients with severe eosinophilic asthma. $N$ Engl J Med. 2014;371 (13):1198-1207. doi:10.1056/NEJMoa1403290

15. Caminati M, Cegolon L, Vianello A, et al. Mepolizumab for severe eosinophilic asthma: a real-world snapshot on clinical markers and timing of response. Expert Rev Respir Med. 2019;13(12):1205-1212. doi:10.1080/17476348.2019.1676734

16. Montero-Perez O, Contreras-Rey MB, Sanchez-Gomez E. Effectiveness and safety of mepolizumab in severe refractory eosinophilic asthma: results in clinical practice. Drugs Context. 2019;8:212584. doi:10.7573/dic. 212584

17. Strauss RA, Jawhari N. Mepolizumab in the treatment of severe eosinophilic asthma: results from a physician in the field. Ann Allergy Asthma Immunol. 2018;121(1):121-123. doi:10.1016/j. anai.2018.04.016

18. JP OH L, Bogart M, Packnett ER, Manjelievskaia J, Bell CF, Hahn B. Real-world effectiveness of mepolizumab in patients with severe asthma: an examination of exacerbations and costs. $J$ Asthma Allergy. 2020;13:77-87. doi:10.2147/JAA.S236609

19. Liu D, Ahmet A, Ward L, et al. A practical guide to the monitoring and management of the complications of systemic corticosteroid therapy. Allergy Asthma Clin Immunol. 2013;9(1):30. doi:10.1186/1710-1492-9-30

20. Mundell L, Lindemann R, Douglas J. Monitoring long-term oral corticosteroids. BMJ Open Qual. 2017;6(2):e000209. doi:10.1136/bmjoq2017-000209 
21. Waljee AK, Rogers MA, Lin P, et al. Short term use of oral corticosteroids and related harms among adults in the United States: population based cohort study. BMJ. 2017;357:j1415. doi:10.1136/bmj.j1415

22. Pavord ID, Beasley R, Agusti A, et al. After asthma: redefining airways diseases. Lancet. 2018;391(10118):350-400. doi:10.1016/ S0140-6736(17)30879-6
23. Quan H, Li B, Couris CM, et al. Updating and validating the Charlson comorbidity index and score for risk adjustment in hospital discharge abstracts using data from 6 countries. Am J Epidemiol. 2011;173 (6):676-682. doi:10.1093/aje/kwq433

\section{Publish your work in this journal}

The Journal of Asthma and Allergy is an international, peer-reviewed open-access journal publishing original research, reports, editorials and commentaries on the following topics: Asthma; Pulmonary physiology; Asthma related clinical health; Clinical immunology and the immunological basis of disease; Pharmacological interventions and

Submit your manuscript here: https://www.dovepress.com/journal-of-asthma-and-allergy-journal new therapies. The manuscript management system is completely online and includes a very quick and fair peer-review system, which is all easy to use. Visit http://www.dovepress.com/testimonials.php to read real quotes from published authors. 